\title{
Evaluation of a 3D diamond detector for medical radiation dosimetry
}

DOI:

10.1088/1748-0221/12/01/P01003

\section{Document Version}

Accepted author manuscript

Link to publication record in Manchester Research Explorer

\section{Citation for published version (APA):}

Kanxheri, K., Servoli, L., Oh, A., Munoz Sanchez, F., Forcolin, G., Murphy, S., Aitkenhead, A., Moore, C. J., Morozzi, A., Passeri, D., Bellini, M., Corsi, C., Lagomarsino, S., \& Sciortino, S. (2017). Evaluation of a 3D diamond detector for medical radiation dosimetry. Journal of Instrumentation, 12. https://doi.org/10.1088/1748-

0221/12/01/P01003

\section{Published in:}

Journal of Instrumentation

\section{Citing this paper}

Please note that where the full-text provided on Manchester Research Explorer is the Author Accepted Manuscript or Proof version this may differ from the final Published version. If citing, it is advised that you check and use the publisher's definitive version.

\section{General rights}

Copyright and moral rights for the publications made accessible in the Research Explorer are retained by the authors and/or other copyright owners and it is a condition of accessing publications that users recognise and abide by the legal requirements associated with these rights.

\section{Takedown policy}

If you believe that this document breaches copyright please refer to the University of Manchester's Takedown Procedures [http://man.ac.uk/04Y6Bo] or contact uml.scholarlycommunications@manchester.ac.uk providing relevant details, so we can investigate your claim.

\section{OPEN ACCESS}




\title{
Evaluation of a 3D diamond detector for medical radiation dosimetry
}

\author{
K. Kanxheri ${ }^{*}, 1,2$, L. Servoli ${ }^{1,2}$, A. Oh ${ }^{3}$, F. Munoz Sanchez ${ }^{3}$, G. T. Forcolin ${ }^{3}$, S. A. \\ Murphy $^{3}$, A. Aitkenhead ${ }^{4,5}$, C. J. Moore ${ }^{4,5}$, A. Morozzi ${ }^{1,2}$, D. Passeri ${ }^{1,2}$, M. Bellini ${ }^{6}$, C. \\ Corsi $^{7}$, S. Lagomarsino $0^{8,9}$, S. Sciortino ${ }^{8,9}$ \\ ${ }^{1}$ National Institute of Nuclear Physics (INFN) Perugia \\ ${ }^{2}$ University of Perugia \\ ${ }^{3}$ University of Manchester \\ ${ }^{4}$ The Christie NHS Foundation Trust \\ ${ }^{5}$ Manchester Academic Health Science Centre \\ ${ }^{6}$ INO-CNR Florence \\ ${ }^{7}$ LENS Florence \\ ${ }^{8}$ National Institute of Nuclear Physics (INFN) Florence \\ ${ }^{9}$ University of Florence \\ E-mail: keida.kanxheri@pg.infn.it
}

\begin{abstract}
Synthetic diamond has several properties that are particularly suited to applications in medical radiation dosimetry. It is tissue equivalent, not toxic and shows a high resistance to radiation damage, low leakage current and stability of response. It is an electrical insulator, robust and realizable in small size; due to these features there are several examples of diamond devices, mainly planar single-crystalline chemical vapor depositation (sCVD) diamond, used for relative dose measurement in photon beams. Thanks to a new emerging technology, diamond devices with 3-dimensional structures are produced by using laser pulses to create graphitic paths in the diamond bulk. The necessary bias voltage to operate such detector decreases considerably while the signal response and radiation resistance increase. In order to evaluate the suitability of this new technology for measuring the dose delivered by radiotherapy beams in oncology a 3D polycrystalline (pCVD) diamond detector designed for single charged particle detection has been tested and the photon beam profile has been studied. The good linearity and high sensitivity to the dose observed in the 3D diamond, opens the way to the possibility of realizing a finely segmented device with the potential for dose distribution measurement in a single exposure for small field dosimetry that nowadays is still extremely challenging.
\end{abstract}

KEYWORDS: 3D pCVD diamond detector; graphitic electrodes; dosimetry; radiation therapy.

\footnotetext{
* Corresponding author.
} 


\section{Contents}

1. Introduction $r$

2. Device fabrication $\quad 3$

2.1 Leakage Current $\quad 4$

3. Experimental setup $\quad 5$

4. Results $\quad 6$

4.1 Signal time structure $\quad 6$

4.2 I-V Characteristics $\quad 6$

$\begin{array}{ll}4.3 \text { Linearity } & 7\end{array}$

4.4 Normalized charge response $\quad 8$

4.5 Energy dependence $\quad 9$

$\begin{array}{ll}4.6 \text { Beam profile } & 10\end{array}$

5. Small field dosimetry $r$

6. Conclusions $r$

Acknowledgments $\quad 12$

Footnotes $\quad 12$

References $\quad 12$

\section{Introduction}

Research developments in the field of dosimetry for radiation therapy have led to various studies on the use of diamond as a dosimeter because of its many properties compatible with the required ones for this kind of application. Properties of diamond, such as tissue-equivalence, high radiation sensitivity and high resistance to radiation damage and are very advantageous for clinical dosimetry [1-4]. Types of diamond used have ranged from natural to synthetic diamond like polycrystalline CVD and high quality single crystal CVD [5-6], but regardless of this wide range of choices there are limitations that prevent widespread commercialization of detectors made with either natural or synthetic diamond. In diamond material, defects and impurities are present and they strongly influence the lifetime of charge carriers, generating a slow dynamic response and affecting the reproducibility and time stability [7]. In the last decade most of these limitations have been overcome but the dimensions of the detector's sensitive volume and the electrode geometry are still crucial to the solution of the dosimetric issues observed in small photon beams with diamond detectors [8-9]. Recently a new technology to create graphitic 
paths in the diamond bulk has emerged [10-13] allowing to create 3-dimensional structures like the 3-D silicon detectors [14]. It consists of the arrangement of thin, cylindrical electrodes only a few tens of microns apart, perpendicular to the sensor surface, in such a way that the generated charge inside the material is confined within the single cell delimited by the electrodes at its vertices and is collected by a single electrode at the center of the cell (Fig. 1). In a conventional planar radiation detector the track of the particles and the path followed by each generated charge drifting inside the active volume have about the same length, of the order of the sensor thickness. If the material has high defect density, the charge collection efficiency is limited, at most, to the ratio between the mean capture length and the sensor thickness [15] (Fig. 2, left).
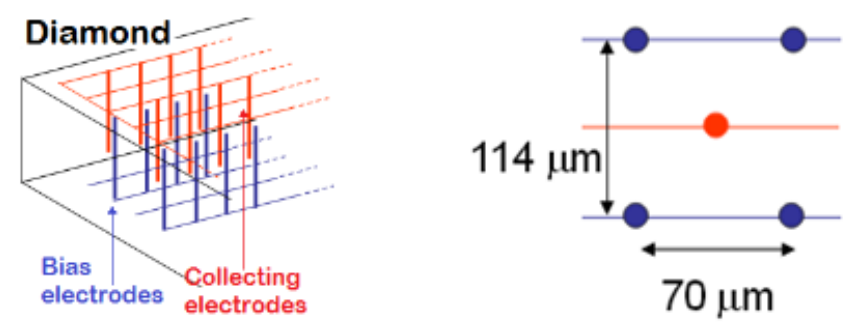

Figure 1. 3D diamond detector concept (left) and the schematic of the single 3-D cell delimited by the electrodes at its vertices (right). Reprinted from [13].

One parameter for optimizing detector performance is the selection of an appropriate bias voltage. The so called "3D" concept allows the employment of a lower bias voltage at which the saturation charge velocity is reached (full polarization voltage) and faster detector response, due to the decreased distance between the polarizing electrodes. In order to allow the collection of more charge, even from lower quality diamond, the distance between electrodes must be smaller than the charge collection distance (CCD). A smaller electrodes distance increases the amount of generated charge that could be collected before trapping giving rise to a higher charge collection efficiency with a lower saturation voltage (Fig. 2, right) [16].The full collection efficiency of $\beta$-induced signals for a 3D diamond detector fabricated in a $500 \mu \mathrm{m}$ thick diamond substrate is reached at voltages about ten times lower than for a $2 \mathrm{D}$ sensor made of the same diamond substrate [13]. This is an important advantage for 3D detectors because high full collection efficiency voltages are generated by high accuracy high voltage source meters which are very expensive and usually bulky. This makes the necessity for high bias voltages a nonfavorable condition for high performance dosimeters. Also the leakage current of the 3D diamond is on average one order of magnitude lower, even after radiation damage [17]. The improved result is a higher and a faster diamond detector response applying a lower bias voltage.

It has also been proven that 3D diamond detectors operate more efficiently in hard radiation environments. Due to the much shorter electrodes distance, the 3D diamond detector charge collection efficiency is less deteriorated by the radiation damage of the diamond material [17]. The pulsed laser technique yields devices with high spatial granularity, (cell sizes of less than 1 $\mathrm{mm}$ ) allowing measurement of the spatial dose distribution. 


\section{Device fabrication}

For the reader's convenience we report here an overview of the fabrication process fully described in ref [13]. The present approach to 3D diamond detectors fabrication is based on a pulsed laser technique which creates graphitic structures in the diamond bulk. A 3D pCVD diamond detector was produced in collaboration with the National Institute of Nuclear Physics (INFN) and the European Laboratory for Nonlinear Spectroscopy (LENS) of Florence.
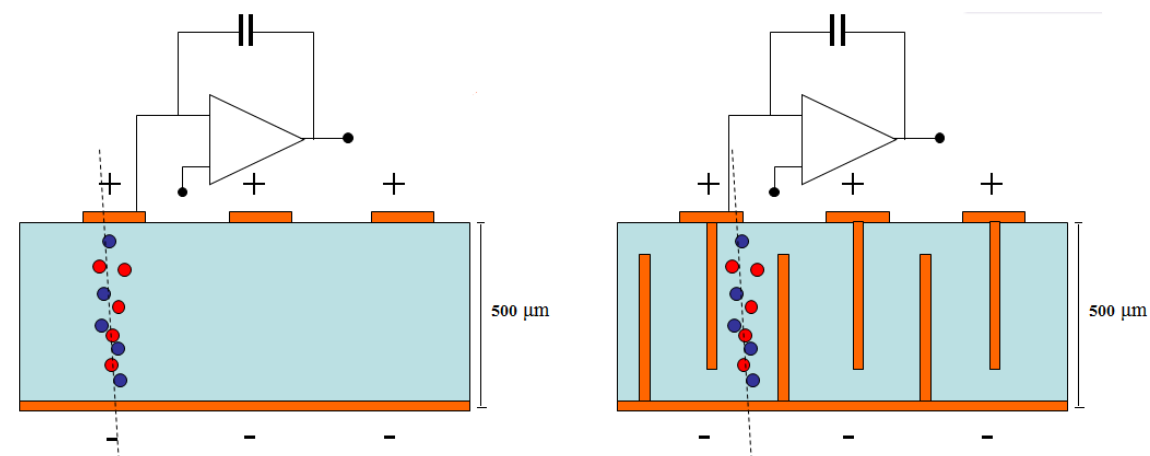

Figure 2. Planar diamond detector (left), 3D diamond detector (right). The light blue structures represent the diamond substrates and the red ones the graphitic electrodes.

The diamond sample was irradiated by a laser beam which was moved from the surface of the substrate to the direction of its thickness $(500 \mu \mathrm{m}$, Fig. 2, right), leaving a graphitic conductive path in the diamond bulk (Fig. 3, left). The characteristics of the graphitic material depend, principally on the laser intensity, wavelength and pulse duration, ranging from the femtoseconds to the nanoseconds.

In this case a femtosecond laser was used to create two combs of graphitic electrodes engraved on the opposite side of the sample, in a way that the projection of the first one onto the opposite side results interdigitated with the other one, stopping each column at about $80 \mu \mathrm{m}$ from the opposite surface (Fig. 1, left and Fig.2, right) [13].

The 3D fabricated device is an all-carbon detector with two combs of graphitic electrodes intended to support ground and bias polarization respectively. The diamond sample is then connected to the read out electronics by placing a small amount of silver paste in the outermost part of the sensor surface as shown in fig $3 \mathrm{~b}$ ).
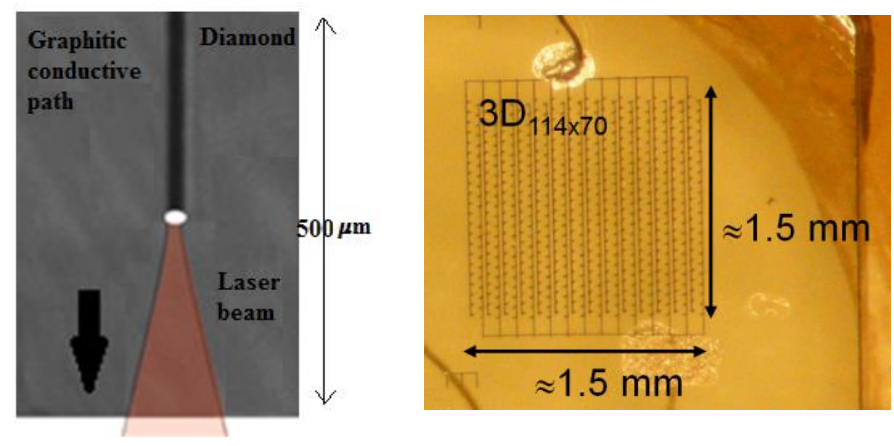

Figure 3. Pulsed laser technique (left), the fabricated prototype (right). Figure 3 (right) reprinted from [13]. 
The distance between the electrodes was chosen so as to be lower than the charge collection distance $(\mathrm{CCD} \approx 150 \mu \mathrm{m})$. The resistivity of the graphitic material made with the fs-laser source was $800 \mathrm{~m} \Omega \mathrm{cm}$. The resulting structure has arrays of graphitic columns of about $10 \mu \mathrm{m}$ in diameter forming cells of $114 \times 70 \mu \mathrm{m}$ with a thickness of $0.5 \mathrm{~mm}$ for a single cell sensitive volume of $0.004 \mathrm{~mm}^{3}$ and a total diamond sensitive volume of about $1.1 \mathrm{~mm}^{3}$ (Fig. 3, right).

The fast evolution of the pulsed laser technique allows fabrication of 3D diamond devices with a repeatable high precision intra-electrode distance, electrode diameter and elementary cell geometry. This means building 3D cells with high precision and substantially reduce the variability between their sensitive volumes. Because of their small dimensions and very precise sensitive volumes [18], the "3D" detectors are potentially applicable to small field dosimetry. For such fields, the dose changes noticeably across the detector because the size of standard detectors is large compared to the beam dimensions. Consequently the signal is subject to the volume effect provoking the underestimation of dose in the field and the overestimation of penumbra width [19]. This makes the detector size and the sensitive volume a crucial requirement for this kind of dosimetry.

Furthermore, since the metal electrodes necessary in the traditional planar diamond dosimeters, which can significantly affect the detector response [20], have been replaced by the graphitic electrodes and the dosimeter is made of carbon only, the need for correction factors is sharply reduced.

\subsection{Leakage Current}

In order to verify the quality of the graphitic columns, IV curves were measured (Fig. 4). The detector is not currently equipped with a read out system able to read each cell individually because it was not designed for dosimetric purposes. Using a Keithley source meter 2410 the detector has been polarized and the output current is obtained by reading in parallel all the collecting graphitic columns.

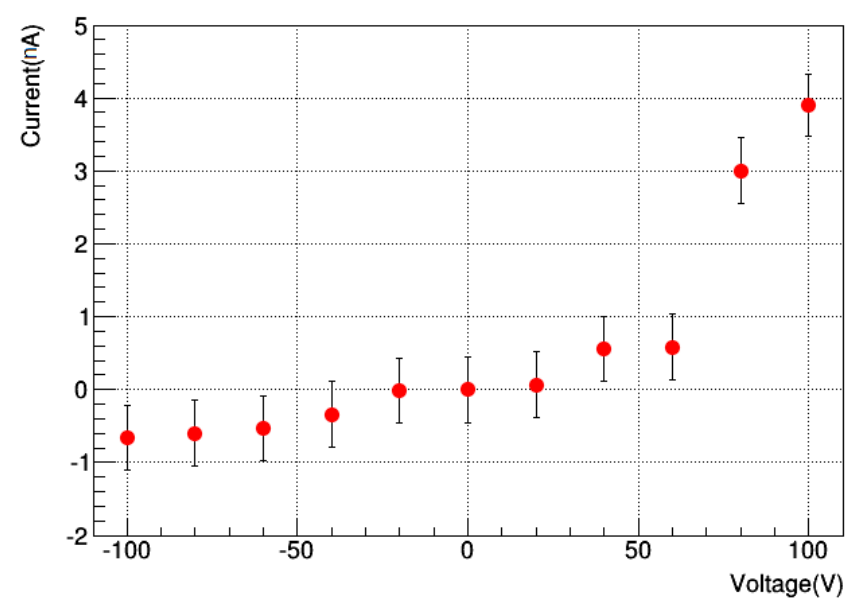

Figure 4. 3D Diamond detector leakage current for voltages between -100 and 100 volts.

The leakage currents at voltages lower than $60 \mathrm{~V}$ are of the order of some hundreds of pA at most, and the characteristic is nearly symmetric with respect to the polarization sign. For higher voltages, the current increases, while the signal shows short time discharge-like erratic oscillations. Such behavior can be attributed to the relatively high electric field $(\approx 10 \mathrm{~V} / \mu \mathrm{m})$ 
near the graphitic columns, in a region where the laser beam has possibly damaged the diamond bulk. The asymmetric characteristics arising at high voltages could be due to slight different diameters of the columns fabricated on the two sides of the diamond during different fabrication sessions. Hence, the voltages used to bias the device during the measurements were always below $60 \mathrm{~V}$.

\section{Experimental setup}

The response of the diamond detector was studied by irradiating it with photons $(6,10 \mathrm{MV})$ at $400 \mathrm{~Hz}$ produced by a medical linear accelerator (Elekta Synergy Sband) with $10 \times 10 \mathrm{~cm}^{2}$ field at the Christie's Hospital in Manchester. Conventionally, the energy of diagnostic and therapeutic X-rays is expressed in MV. This voltage is the maximum electric potential used by the linear accelerator to produce the photon beam. The beam is made up of a spectrum of energies, where the maximum energy is approximately equal to the beam's maximum electric potential times the electron charge [21].The pulse rate of $400 \mathrm{~Hz}$ corresponds to $400 \mathrm{MU} \mathrm{min}^{-1}$ and that different dose rates are achieved by changing the pulse repitition rate while the dose per pulse remains constant.

The radiation beam was, in all the cases, perpendicular to the diamond surface and the sourcedevice distance was $1 \mathrm{~m}$. The response dependence with the dose rate was investigated for a total dose of $1000 \mathrm{MU}$ and dose rates of $290 \mathrm{MU} \mathrm{min}{ }^{-1}, 465 \mathrm{MU} \mathrm{min}{ }^{-1}, 585 \mathrm{MU} \mathrm{min}{ }^{-1}$, where 1 $\mathrm{MU} \approx 0.694 \mathrm{cGy}$ for the above conditions. The photon beam profile in the plane perpendicular to beam propagation was measured by moving the field laterally relative to the detector. The obtained beam profile was compared to a profile measured using Gafchromic EBT2 film which was placed in the same positions as the diamond sensor, and scanned after irradiation. The sensor under test is biased with a full polarization voltage of $-80 \mathrm{~V}$ and the readout system is the same of section 2 . The test facilities and the setup are shown in figure 5 (center and right respectively).
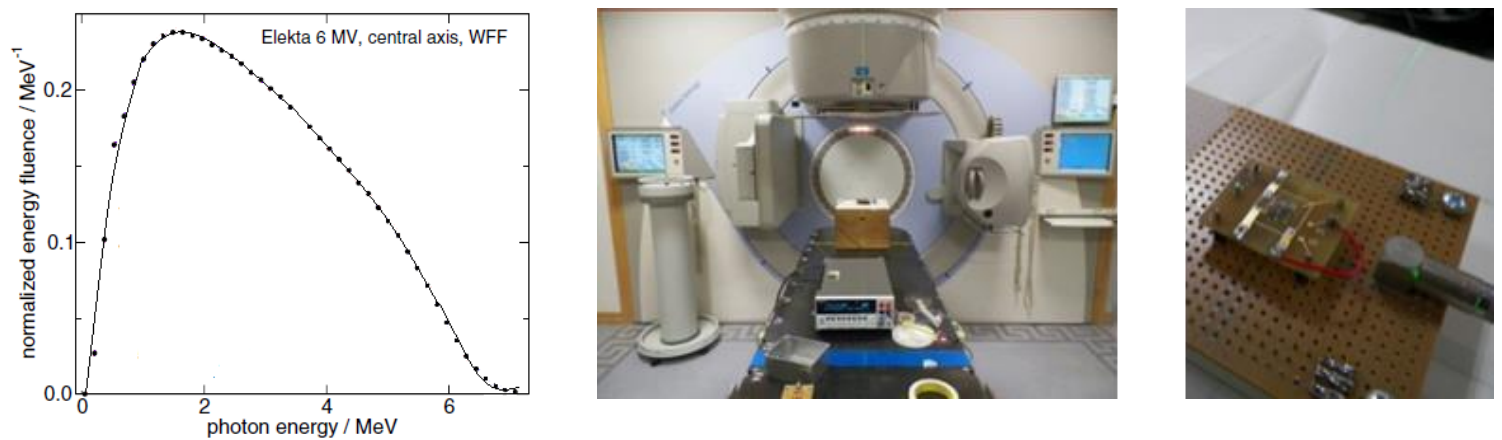

Figure 5. Photon energy spectra of Elekta linac (left), Experimental set-up at the Christie's hospital (center), 3D diamond detector during measurements (right). 


\section{Results}

\subsection{Signal time structure}

To observe the temporal response of the current as a function of the operating voltage, the dose rate was fixed at $585 \mathrm{MU} \min ^{-1}\left(4 \mathrm{~Gy} \mathrm{~min}^{-1}\right)$, the beam at $6 \mathrm{MV}$ for a total delivered dose of $1000 \mathrm{MU}$ (6.9 Gy). Five different bias voltages have been used: $-40 \mathrm{~V},-60 \mathrm{~V},-80 \mathrm{~V},-100 \mathrm{~V}, 60 \mathrm{~V}$ (Fig. 6).

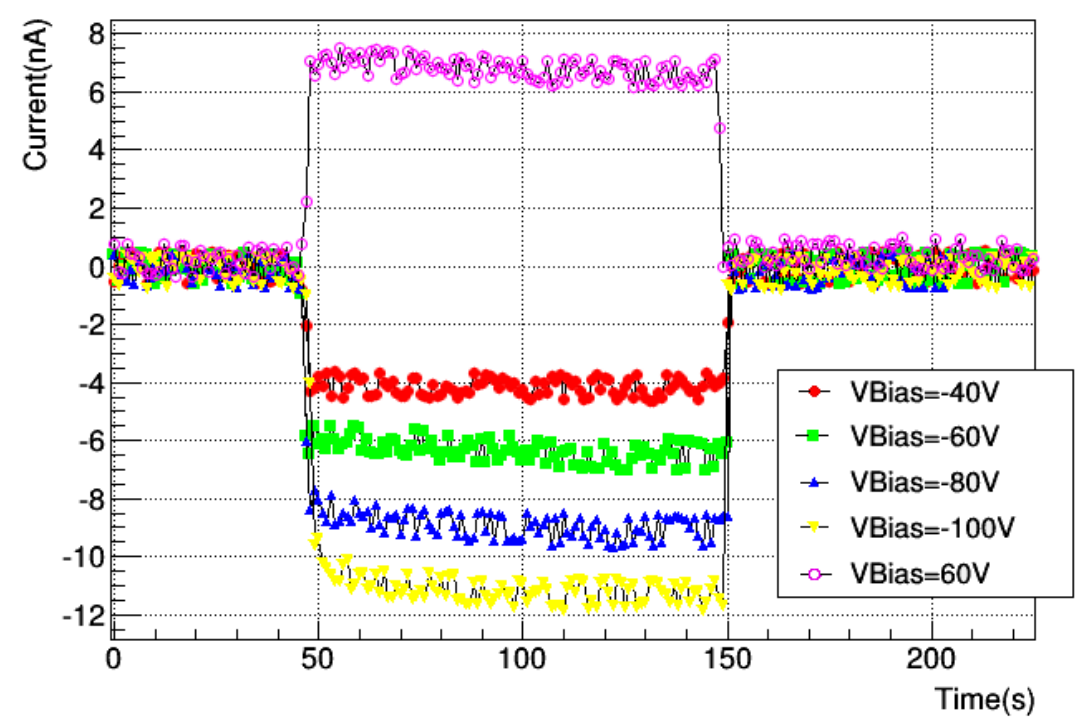

Figure 6. Temporal response in current as a function of the operating voltage during irradiation with 6 $\mathrm{MV}$ photon beam and a dose rate of $585 \mathrm{MU} \mathrm{min}^{-1}$.

In order to reach the response stability of most synthetic diamond detectors a pre-irradiation time is often required, which can vary with the characteristics of the diamond material, as reported in the literature [22-23]. The device under test was pre-irradiated with a dose of $5 \mathrm{~Gy}$ before any measurement was taken. When the photon beam was switched off, the current returned to the dark current value within 1 second. This time was compatible with the instrumental temporal resolution. The detector was sensitive to the presence of the beam for all the operating voltages and the level of the leakage current before and after irradiation remained the same within the measuring instrument uncertainty.

\subsection{I-V Characteristics}

Figure 7 shows the value of the 3D diamond detector current versus the operating voltage during an irradiation by $6 \mathrm{MV}$ photon beam with a dose-rate of $585 \mathrm{MU} \mathrm{min}^{-1}\left(4 \mathrm{~Gy} \min ^{-1}\right.$ ). As reported in section 2.1 the detector leakage current in absence of the photon beam for each bias voltage was measured. The resulting I-V data in absence and in presence of the photon beam were fit to a linear law and the leakage current function was subtracted from the detector response. In order to evaluate the linearity of the measured current values as a function of the bias voltage, the response of the detector at $80 \mathrm{~V}$ was excluded from the fit, because of the measured high leakage current. The absence of a significant deviation from the linear fit of the 
leakage current at bias voltages lower than $60 \mathrm{~V}$ is encouraging, as it eliminates the need for an additional measurement session for each bias voltage before irradiating the sensor with the medical radiation beam, hence a single measurement for dose evaluation. The error bars related to the detector output current during each measurement are given by a number of uncertainties related to the photon beam, to the measuring instrument and to the detector read out system. Comparing measurements with and without the photon beam (red and green markers in figure 7 , respectively), it is possible to notice that the error bars are almost the same $(\approx 0.5 \mathrm{nA})$. This indicates that the uncertainty coming from the oscillations of the measuring instrument and the read out system is dominating any other uncertainty due to the photon beam.

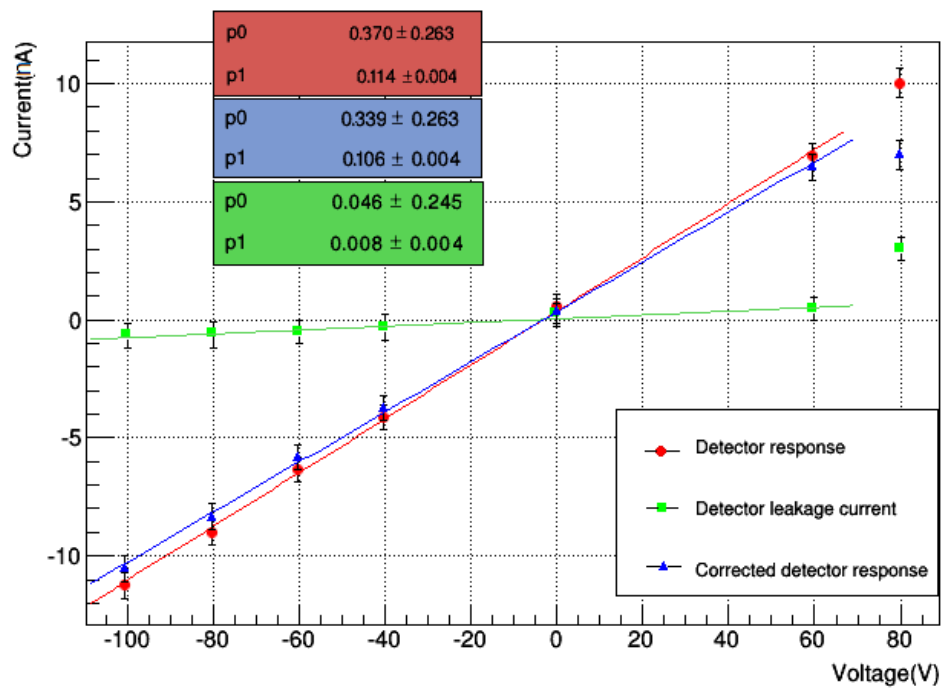

Figure 7. Detector leakage current (green line), current-voltage characteristics of the $3 \mathrm{D}$ diamond when irradiated with $6 \mathrm{MV}$ photon beam and a dose rate of $585 \mathrm{MU} \mathrm{min}^{-1}$ (red line), current-voltage characteristics of the 3D diamond when irradiated with $6 \mathrm{MV}$ photon beam and a dose rate of $585 \mathrm{MU}$ $\mathrm{min}^{-1}$ after subtraction of the leakage current (blue line).

\subsection{Linearity}

The output signal of the detecor is an electric current so in principle it measures dose rates. Linear detector response is important because it guarantees independence of the dose from the dose rate. To this purpose the detector was biased with $-80 \mathrm{~V}$ and tested for dose rate dependence. The detector response was measured for 3 different dose rates: $290 \mathrm{MU} \min ^{-1}(2 \mathrm{~Gy}$ $\min ^{-1}$ ), $465 \mathrm{MU} \min ^{-1}\left(3.2 \mathrm{~Gy}_{\min ^{-1}}\right.$ ), and $585 \mathrm{MU} \min ^{-1}\left(4 \mathrm{~Gy} \min ^{-1}\right)$.

It is common to fit this relationship to the empirical expression of Fowler [24]:

$$
\mathbf{I}=\mathbf{I}_{\mathrm{dark}}+\mathbf{K}^{*} \mathbf{D}^{\mathbf{\Lambda}}
$$

where $\mathrm{I}$ is the detector signal, $\mathrm{I}_{\mathrm{dark}}$ the leakage current, $\mathrm{K}$ is a parameter for the detector sensitivity, $\mathrm{D}$ is the dose rate and $\Delta$ is the correlation factor for the observed values, known also as linearity indicator. We obtained $\Delta=0.997 \pm 0.003$. A correlation factor so close to 1 , indicates a linear dependence between current response and dose rate values (Figure 8 left, top), hence a simple linear fit function is applied. The Figure 8 left, bottom, reports the residuals between the predicted values and the measured ones (deviations from linear fit). For each point 
of measurement there is always a deviation from linearity within $1 \%$, indicating negligible dose rate dependence.

By integrating the detector currents at constant dose rates the charge response of the diamond detector under irradiation has also been studied (Fig.8, right). The measurements were done at $585 \mathrm{MU} \mathrm{min}{ }^{-1}\left(4 \mathrm{~Gy} \mathrm{~min}^{-1}\right.$ ) and the different doses have been obtained with photon irradiation lasting respectively $10 \mathrm{~s}, 20 \mathrm{~s}, 30 \mathrm{~s}, 40 \mathrm{~s}$, and $100 \mathrm{~s}$.
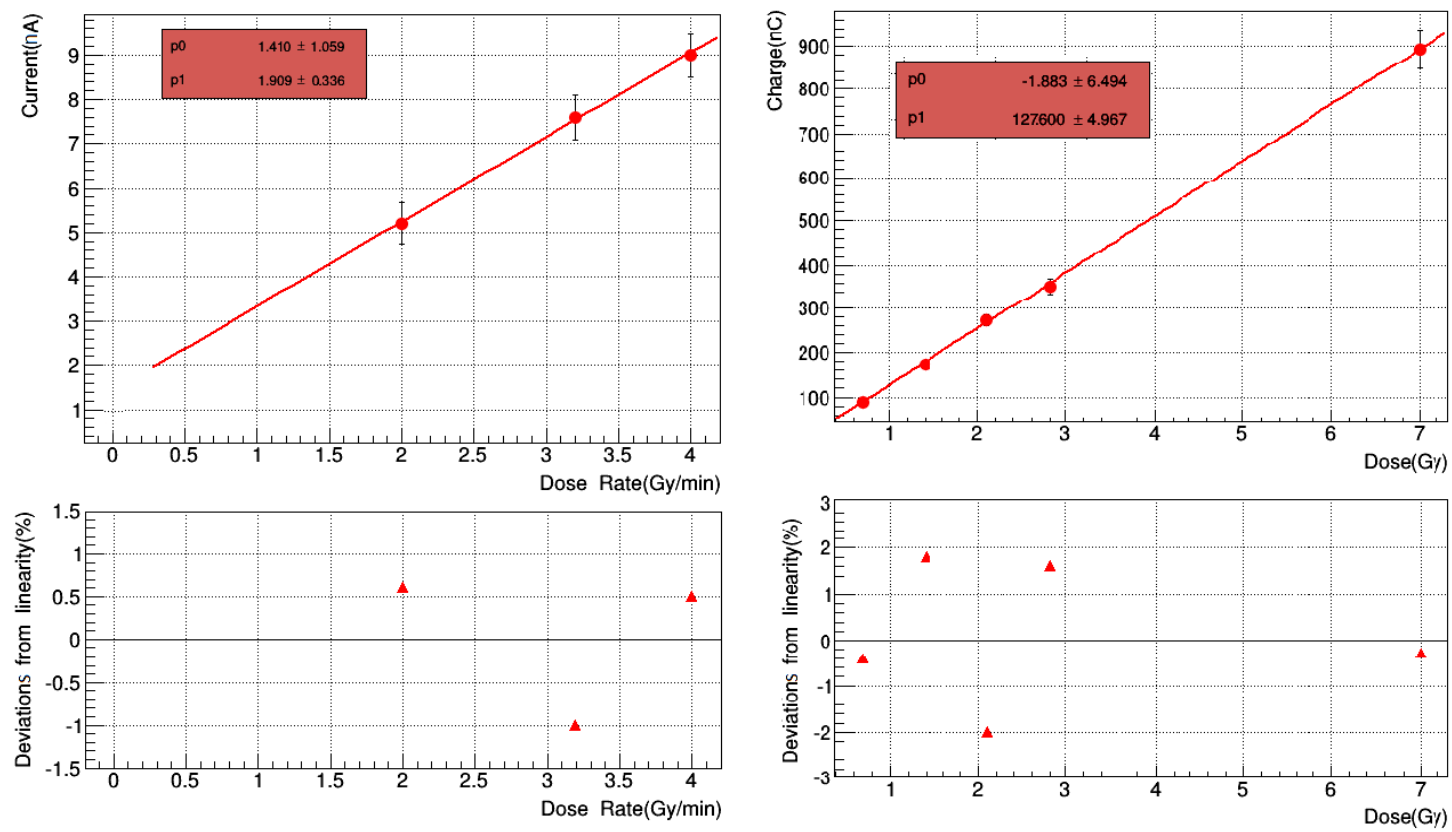

Figure 8. Linearity with dose rate for 3D diamond during irradiation with $6 \mathrm{MV}$ photons and operating voltage of $-80 \mathrm{~V}$ (left, top). Residuals between the predicted current values and the measured ones (deviations from linear fit) (left, bottom). Linearity with dose for 3D diamond during irradiation with 6 MV photons and operating voltage of $-80 \mathrm{~V}$ (right, top). Residuals between the predicted charge values and the measured ones, (right, bottom)

The correlation factor in this case is $0.995 \pm 0.003$ indicating linearity of the charge response in function of dose (Fig. 8 right, top). The deviations from the linear fit are always within $2 \%$ (Fig. 8 right, bottom). This value is an indicator of the good time stability of the detector response.

\subsection{Normalized charge response}

The diamond detector charge response normalized to the dose rate was found after the beam and detector stabilized at $585 \mathrm{MU} \mathrm{min} \mathrm{m}^{-1}\left(4 \mathrm{~Gy} \mathrm{~min}^{-1}\right)$ and represented as a function of the bias voltage in figure 9. A linear correlation is observed in the range -100 to $60 \mathrm{~V}$ (see the linear fit in figure 9, top). The response values varied over the range of tested voltages from about -150 to $90 \mathrm{nC} \mathrm{Gy}^{-1}$. These are high sensitivity values if compared for example with the sensitivity of the PTW 30013 Waterproof Farmer Chamber with $6 \mathrm{~mm}^{3}$ sensitive volume (Radiation Products Design, Inc) that is about $20 \mathrm{nC} \mathrm{Gy}^{-1}$. At $-100 \mathrm{~V}$ the detector shows the highest sensitivity $(-150$ $\mathrm{nC} \mathrm{Gy}^{-1}$ ) but also a non-negligible and partially unstable leakage current, hence the operating voltage was fixed at $-80 \mathrm{~V}$. 

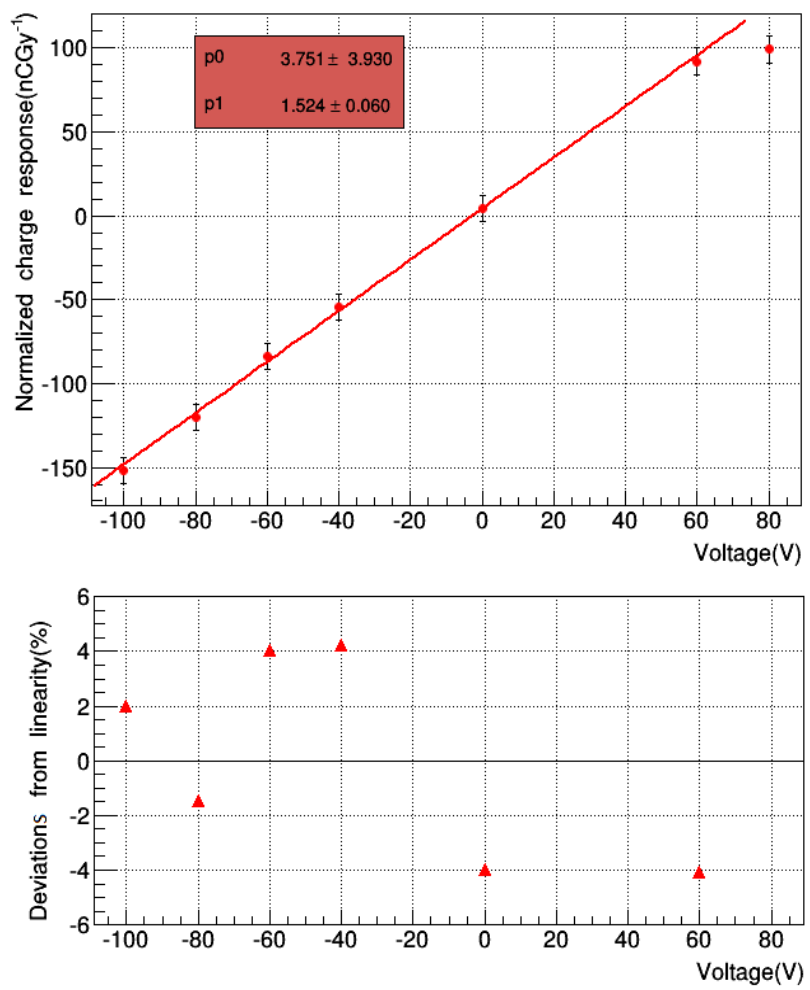

Figure 9. 3D diamond charge response normalized to the dose rate versus bias voltage. The response increases with increasing the bias voltage (top). Deviations from the linear fit for each point of measurement (bottom).

\subsection{Energy dependence}

Ideally, the energy response should be flat, i.e., the system calibration should be independent of energy [25]. In reality, since the dosimetry systems are calibrated at a specified radiation beam energy and used over a much wider energy range, the variation of the response of a dosimetry system (called energy dependence) should be corrected for. The current response of the 3D diamond detector was determined for photon beams of two different energies. The diamond detector response does not change significantly, about $3 \%$ of variation from $6 \mathrm{MV}$ to $10 \mathrm{MV}$.

\begin{tabular}{l|ccc}
\hline & No beam & $6 \mathrm{MV}$ & $10 \mathrm{MV}$ \\
\hline Mean value (nA) & 0.605 & 90.740 & 94.630 \\
Uncertainty (nA) & \pm 0.332 & \pm 0.364 & \pm 0.341 \\
\hline
\end{tabular}

Table 1. 3D detector current response versus the photon beam energy with a dose rate of $585 \mathrm{MU} \mathrm{min}^{-1}$ $\left(4 \mathrm{~Gy} \mathrm{~min}^{-1}\right.$ ) 
Therefore it can be said that the diamond detector shows no energy dependence within the covered range of 6-10 MV photon beams and no correction is needed in this case. The treatment machine was able to emit only photons of $6 \mathrm{MV}$ and $10 \mathrm{MV}$ and it was not possible to carry out measurements outside of these energy conditions.

\subsection{Beam profile}

The beam profile has been obtained both using a GafChromic film and the 3D diamond detector. The beam profile penumbra (distance between the $80 \%$ and $20 \%$ relative dose points) and the field size (width at 50\% relative dose) were then evaluated.

In figure 10 results from the film and diamond detector are compared. There is a good agreement in terms of beam flatness while the profile widths agreed within $1 \%$. The small offset between the beam profiles was likely due to the different irradiation conditions for the diamond device (where the field was scanned across the detector) and the film (where the field was stationary). The precision with which it is possible to determine the distance from the axis is also limited by the diamond detector size.

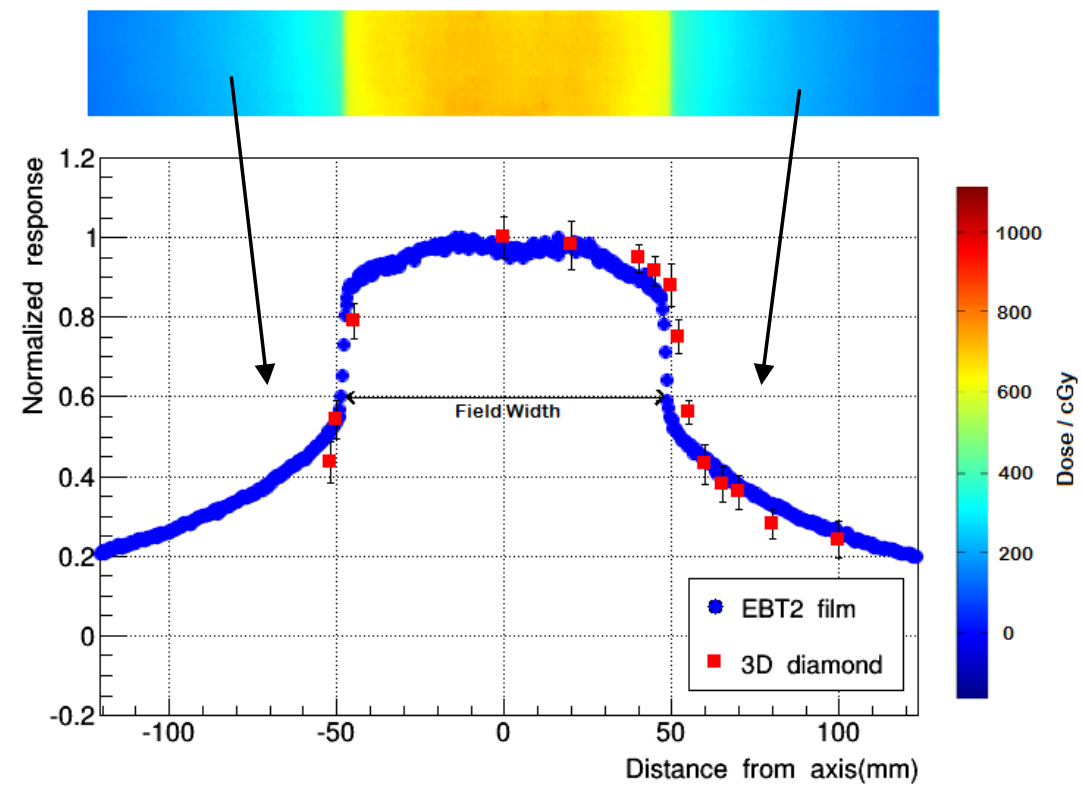

Figure 10. A $10 \times 10 \mathrm{~cm}^{2}$ beam profile measured using the diamond detector (bottom) and the GafChromic film (top). The beam is stabilized at $585 \mathrm{MU} \mathrm{min}^{-1}\left(4 \mathrm{~Gy} \mathrm{~min}^{-1}\right.$ ) with $6 \mathrm{MV}$ and the detector is biased with $-80 \mathrm{~V}$.

The region of major significance in the beam profile is the penumbra, where the absorbed dose falls steeply with increasing distance from the central axis (Fig. 11). This region is especially important for small fields, where a high spatial resolution of the dose measurement is made possible using a small sensitive volume. The penumbra widths measured by the diamond and EBT2 film agreed with each other to within $2 \mathrm{~mm}$, which corresponds to about $3 \%$ of uncertainty. 


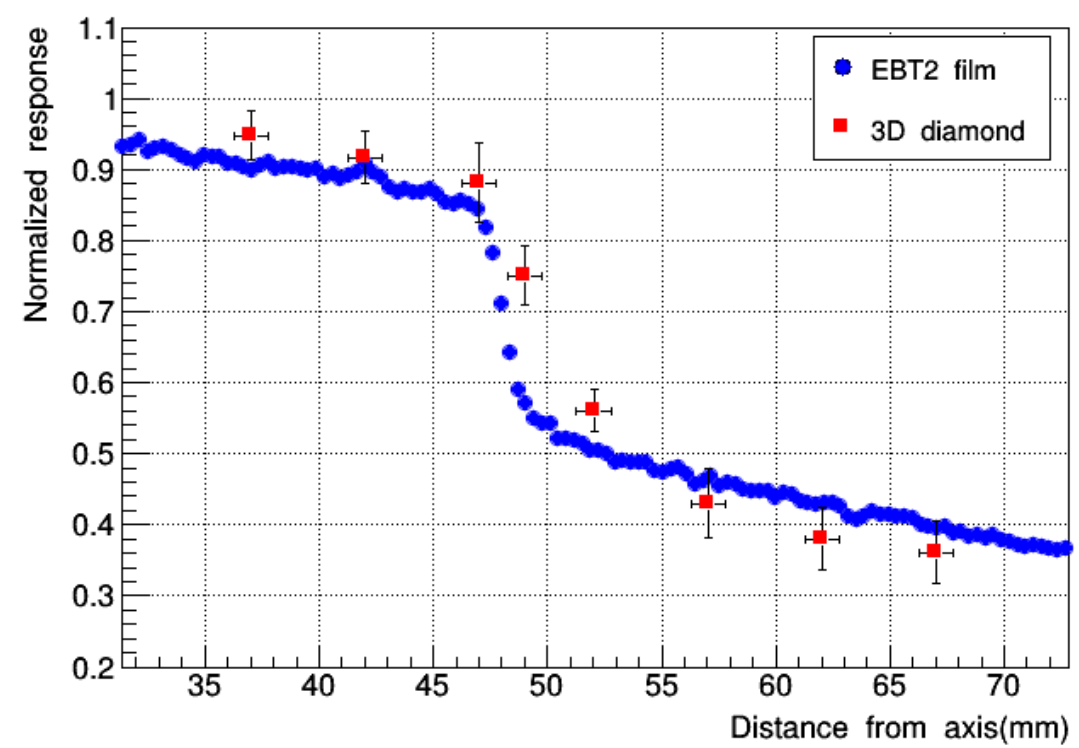

Figure 11. Penumbra region of the $10 \times 10 \mathrm{~cm}^{2}$ beam profile measured using the diamond detector and the GafChromic film.

\section{Small field dosimetry}

A "small field" is generally defined as a field with dimensions smaller than the lateral range of the electrons that contribute to dose [26]. Field sizes below approximately $3 \times 3 \mathrm{~cm}^{2}$ are considered to be small fields in megavoltage photon dosimetry. In these conditions lateral charged particle equilibrium is lost because the size of the field becomes small compared to the range of the dose depositing electrons. Small fields have also a very large percentage of the field made up by penumbra; if the detector is larger than 1/4th of the lateral field dimension, the detector size is large compared to the beam dimensions making volume averaging within the detector problematic [8]. Due to these physical conditions small field dosimetry requires different correction factors related to different characteristics of the detector like the density of the sensitive volume, the presence of extra components like metal electrodes and the volume averaging [27-29]. Recently, in the attempt to reduce as much as possible the need for correction factors, a CVD based radiation detector with a very small sensitive volume has become commercially available from the manufacturer PTW [4]. It shows very good dosimetric characteristics but there is still room for improvement in relation to positional accuracy of each measurement when trying to define the dose field profile. When more measurements are needed to define a dose profile, each uncertainty in the single measurement increases the total uncertainty in the final result. In order to present a solution to this problem, the near future of the 3D diamond dosimeters aims to a new highly segmented larger dosimeter in order to obtain small field profiles in one single shot measurement, reducing the uncertainty of the delivered dose beam profile.

\section{Conclusions}

Results demonstrate the feasibility of 3D diamond devices for dosimetry of clinical megavoltage photon beams, showing a linear dose response with a maximal deviation from 
linearity of $2 \%$ and a high sensitivity of about $80 \mathrm{nC} \mathrm{Gy}^{-1}$ at $-80 \mathrm{~V}$. The diamond detector showed no energy dependence within the covered range of 6-10 MV photon beams, therefore no correction factors are needed. This study showed the capability to use the device in characterization of beam flatness and edge profile showing good agreement with the GafChromic film response in terms of beam flatness and profile widths within $1 \%$. As a reasonable perspective, once the present fabrication technology has been optimized, we can expect that large sensitive area polycrystalline CVD diamond segmented dosimeters will be obtainable (up to $25 \times 25 \mathrm{~mm}^{2}$ ). This would permit precise beam profile determination for small fields $\left(10 \times 10 \mathrm{~mm}^{2}\right)$. Due to the simultaneous measurement of many points not only in the beam shape but also in the penumbra region, a higher accuracy in measurements of very small size field profiles would be possible and the need of using many correction factors will be greatly reduced.

\section{Acknowledgments}

The authors gratefully acknowledge Prof. C. Talamonti for the useful and constructive discussions.

\section{Footnotes}

This work has been partially supported by "Fondazione Cassa di Risparmio Perugia - Progetto 3Dose 2016.0107.021".

\section{References}

[1] C. Buttar, et al., "A study of radiotherapy dosimetery based on diamond grown by chemical vapour deposition", Diamond and Related Materials, 6, 965 - 969, 2000.

[2] A. Fidanzio, et al., "Photon and electron beam dosimetry with a CVD diamond detector", Nucl. Instr. Meth. in Physics Research A: 524 (2004) 115-123.

[3] F. Marsolat, et al., "A new single crystal diamond dosimeter for small beam: comparison with different commercial active detectors", Physics in Medicine and Biology. 2013 Nov 7;58(21):7647-60.

[4] José Manuel, et al., "Properties of a commercial PTW-60019 synthetic diamond detector for the dosimetry of small radiotherapy beams", Physics in Medicine and Biology, Volume 60, Number 2, 2015.

[5] A. Balducci, et al. "Growth and characterization of single crystal CVD diamond film based nuclear detectors", Diamond and Related Materials, 2006, 15(2-3), 292-295.

[6] V. P. Varnin, et al., "The state of art in the growth of diamond crystals and films", Inorganic Materials, 2006, 42, 1; S1-S18.

[7] C. De Angelis et al., "Present limitations of CVD diamond detectors for IMRT applications", Nuclear Instruments and Methods in Physics Research Section A: Accelerators, Spectrometers, Detectors and Associated Equipment, vol. 583, no. 1, pp. 195-203, Dec. 2007.

[8] N. Ade, T.L. Nam, "The influence of detector size relative to field size in small-field photon-beam dosimetry using synthetic diamond crystals as sensors“, doi:10.1016/j.radphyschem.2015.04.005. 
[9] F. Marsolat et al., "Why diamond dimensions and electrode geometry are crucial for small photon beam dosimetry“, J. Appl. Phys. 118, 234507 (2015).

[10] S. Lagomarsino, et al., "Electrical and Raman-imaging characterization of laser-made electrodes for 3D diamond detectors", Diamond and Related Materials, Volume 43, March 2014, Pages 23-28, http://dx.doi.org/10.1016/j.diamond.2014.01.002

[11] V. I. Konov, "Laser in micro and nanoprocessing of diamond materials", Laser Photonics Rev. 6, No.6 739-766 (2012).

[12] A. Oh, et al., "A novel detector with graphitic electrodes in CVD diamond", Diamond \& Related Materials 38 (2013) 9-13.

[13] S. Lagomarsino et al., "Polycrystalline diamond detectors with three-dimensional electrodes", Nuclear Instruments \& Methods in Physics Research, Section A: Accelerators, Spectrometers, Detectors and Associated Equipment 796 42, 2015.

[14] C. Da Vià, et al., "Future trends of 3D silicon sensors", Nuclear Instruments and Methods in Physics Research. Section A: Accelerators, Spectrometers, Detectors, and Associated Equipment, Vol. A731, 2013, p. 201-204.

[15] K. Hecht, "Zum Mechanismus des lichtelektrischen Primärstromes in isolierenden Kristallen", Zeitschrift für Physik, 1932, Volume 77, 3-4, pp235-245.

[16] S. Lagomarsino, et al., "Three-dimensional diamond detectors: Charge collection efficiency of graphitic electrodes”, Applied Physics. Letters 103, 233507 (2013).

[17] S. Lagomarsino et al., "Radiation hardness of three-dimensional polycrystalline diamond detectors", Appl. Phys. Lett. 106, 193509 (2015).

[18] F. Bachmair et al., "A 3D diamond detector for particle tracking", Nuclear Instruments and Methods in Physics Research, A, Volume 786, p. 97-104, 2015.

[19] Laub WU, "The volume effect of detectors in the dosimetry of small fields used in IMRT". Medical Physics. 2003 Mar; 30 (3) : 341-7.

[20] B. Górka, et al., "Influence of electrodes on the photon energy deposition in CVD-diamond dosimeters studied with the Monte Carlo code PENELOPE". Physics in Medicine and Biology. 51: 3607-23, 2006.

[21] W. Di Paul et al., "Cummings Otolaryngology - Head and Neck Surgery”, Elsevier Health Sciences, 28 nov 2014, 6th edition.

[22] M. Marinelli, et al., "Systematic study of pre-irradiation effects in high efficiency CVD diamond nuclear particle detectors". Nuclear Instruments and Methods in Physics Research. Section A: Accelerators, Spectrometers, Detectors, and Associated Equipment, 476(3), 701-705, 2002.

[23] M. Pimpinella et al., "Advances in synthetic diamond detectors dosimetry", Radiotherapy and oncology, 2014, 10.1016/S0167-8140(15)30572-7. 
[24] Fowler J.F, “Solid state electrical conductivity dosimeters”, Radiation Dosimetry Ed Attix F. H and Roesch W. C. (New York Academic), 1966.

[25] "Review of Radiation Oncology Physics: A Handbook for Teachers and Students", chapter 3, pp7576.

[26] R. Alfonso et al., "A new formalism for reference dosimetry of small and nonstandard fields", Medical Physics Letter (2008) 35(11):5179-5186.

[27] D. J. O'Brien et al., "Small field detector correction factors for silicon diode and diamond detectors with circular 6 MV fields derived using both empirical and numerical methods”, Medical Physics, 43, 411, 2016.

[28] H. Bouchard et al., "Detector dose response in megavoltage small photon beams. 1. Theoretical Concepts”. Medical Physics 42, 6033 (2015).

[29] H. Bouchard et al., "Detector dose response in megavoltage small photon beams. 2. Pencil beam perturbation effect”. Medical Physics 42, 6048 (2015). 